\title{
Mechanical Properties of Materials Used For Prosthetic Foot: A Review
}

\author{
K. M. Walke, P. S. Pandure \\ (Department of Mechanical Engineering, M. E. S. College of Engineering, S.P. Pune Unuversity, India)
}

\begin{abstract}
Prosthetic limbs are artificial devices which substitutes missing body parts (limbs).A person may face amputation due to disease or accident. The amputee's limbs are hence replaced by prosthetic limbs. These limbs aren't as multi-functional as natural limbs but try to meet specifications, restricting the performance level. Considering particularly prosthetic foot; selection of devices is based on its characteristics which coincide favorably with the characteristics of human foot. Prosthetic foot are designed in such a way it they meets required value of tensile strength, low density, corrosion resistant, more shear strength, flexible, durable and cost efficient. The above considerations depends on mechanical properties of the material used, Design of the foot and the manufacturing process applied. In this paper, mechanical properties of materials used for prosthetic foot are studied. The various materials used are: Carbon Fiber, Derlin, RCF, Glass fiber, polypropylene, Kevlar etc. This is a comparative study of analysis of material properties by assuming boundary conditions, reaction forces, design consideration and application. The materials studied in this are: Carbon fiber, Reinforced Carbon Fiber, Fiber Glass and Kevlar.
\end{abstract}

Keywords: Carbon Fiber (CF), Fiber Glass, Kevlar, Reinforced Carbon Fiber (RCF), Prosthetic Limbs.

\section{Introduction}

Prosthetic limbs are artificial devices which substitutes missing body parts (limbs) of the amputee. A person may face amputation due to disease or accidents. The amputee's limbs are hence replaced by prosthetic limbs which are designed to meet the performance level of natural human limbs equally providing comfort to the amputee. Prosthetic limbs allow amputees to continue their day to day life. Lower extremity prosthesis provides replacement of lower limbs at varying levels of amputation. Prosthetic foot helps the amputee to walk independently adapting amputee's gait [1]. Prosthetic foot is designed in such a way that they provide comfort to the amputee without compromising the degree of performance. The selection of prosthetic foot is done by considering the properties such as high tensile strength, high compressive strength, low density, high corrosion resistance, high shear strength, flexibility, durability as well as cost efficient and the requirements of the amputee. An ideal prosthetic foot should have dorsiflexion movement of an angle $20^{\circ}$, eversion movement of an angle $20^{\circ}$ (both subscribed at ankle) and $117 \%$ energy return efficiency. These considerations are not only dependent on mechanical properties of the material but also the design of foot and the process of manufacturing. Along with the properties mentioned above, prosthetic feet should also be easily available, easy to repair, easy to replace, adjustable, easy to put on and off and also cost efficient [1].

Due to change in human body over time, due to growth, or change in height, body weight etc.; the prosthetic limbs need to be changed or adjusted time to time. This need of replacement or adjustment of prosthetic devices may become expensive if the material is costly. The commonly used materials for prosthetic foot are Carbon Fiber, Polypropylene, Reinforced Carbon Fiber, Kevlar etc. which meet required properties. According to the requirements and considering physical and mechanical properties of materials, suitable material is selected. Physical and mechanical properties of Carbon Fiber (CF), Fiber Glass, Kevlar and Reinforced Carbon Fiber (RCF) are reviewed further [2].

\section{Carbon Fiber}

Klasson [1] analyzed mechanical properties of Carbon Fiber, Glass Fiber and blend of Glass Fiber and Carbon Fiber for the use in prosthetic foot. Author studied material properties considering the orientation of the fiber laminate. He compared properties of Carbon Fiber, Glass Fiber and blend of Glass Fiber and Carbon Fiber under various tests. He applied bending moment and measured deflection of beam of each material sample. It was found that properly laminated Carbon fiber with continuous and straight fibers was thrice stiffer than Glass Fiber; whereas Glass Fiber structure was five times stiffer than Glass Fiber- Carbon Fiber mix without spending any effort on straightening and orienting the fibers in correct direction. This concludes that if Glass Fiber is correctly used will give excellent result. He put forward an orientation scheme for each layer of fiber which will result in improved tensile and compressive strengths. 


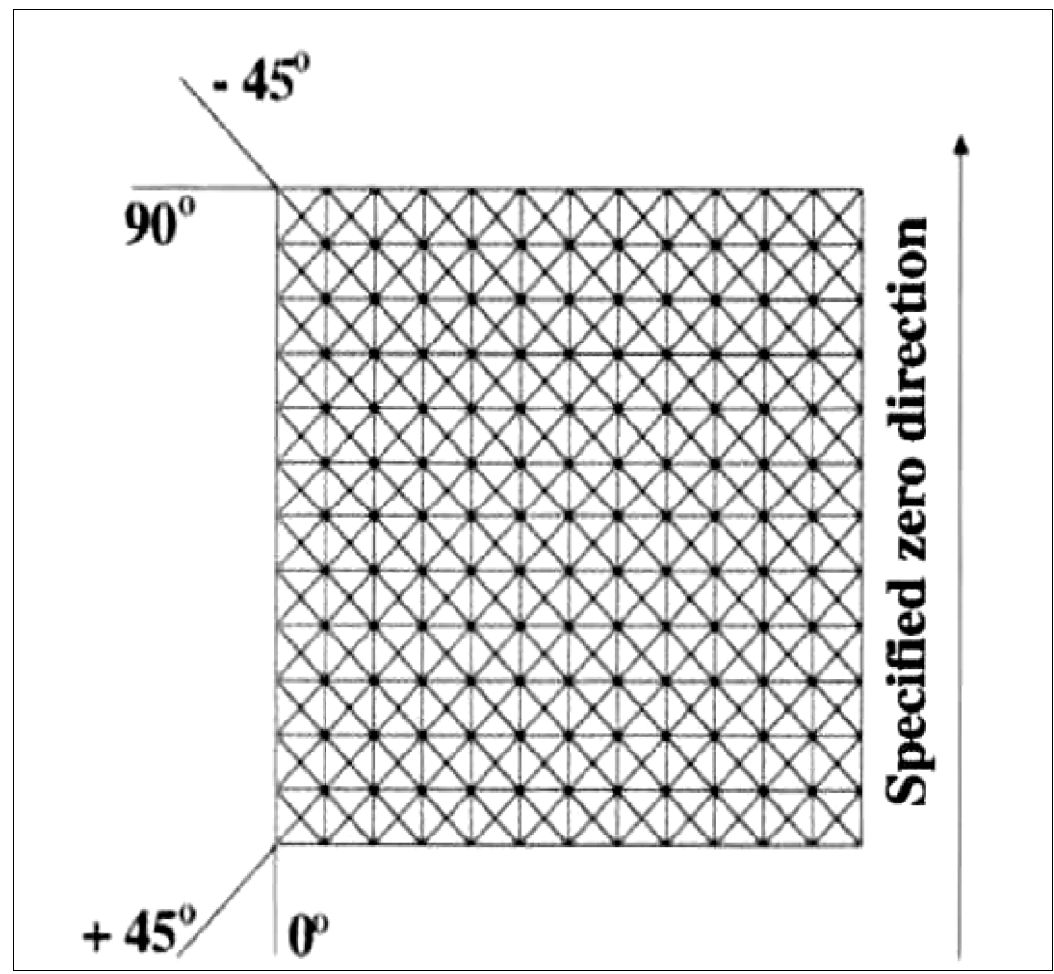

Fig. 1 Orientation of Fiber Laminates.

Proposed orientation scheme:

$$
\begin{aligned}
& 1^{*}+45^{\circ} \\
& 1^{*}-45^{\circ} \\
& 1^{*}+45^{\circ} \\
& 1^{*}-45^{\circ} \\
& 5^{*} 0^{\circ} \\
& 2^{*} 90^{\circ} \\
& \text { Core } \\
& 2^{*} 90^{\circ} \\
& 5^{*} 0^{\circ} \\
& 1^{*}-45^{\circ} \\
& 1^{*}+45^{\circ} \\
& 1^{*}-45^{\circ} \\
& 1^{*}+45^{\circ}
\end{aligned}
$$

Where $+45^{\circ},-45^{\circ}, 0^{\circ}, 90^{\circ}$ represents degree of orientation of fiber laminates. In this structure $0^{\circ}$ fiber handles parallel bending stresses, fibers with $45^{\circ}$ orientation handles shear stress and fibers oriented at $90^{\circ}$ handles perpendicular delaminating stress as shown in Fig. 1.

If there is any hole in continuous fiber interrupts the continuity of fiber. This causes concentration of shear stress in continuous fiber composite and increases risk of delamination. This is not desirable. The sandwich structure proposed by the author is a solution for this problem. As suggested by the author, the alternate orientation of the fiber laminates will result in improvement in shear strength of whole structure as shown in Fig. 2. 


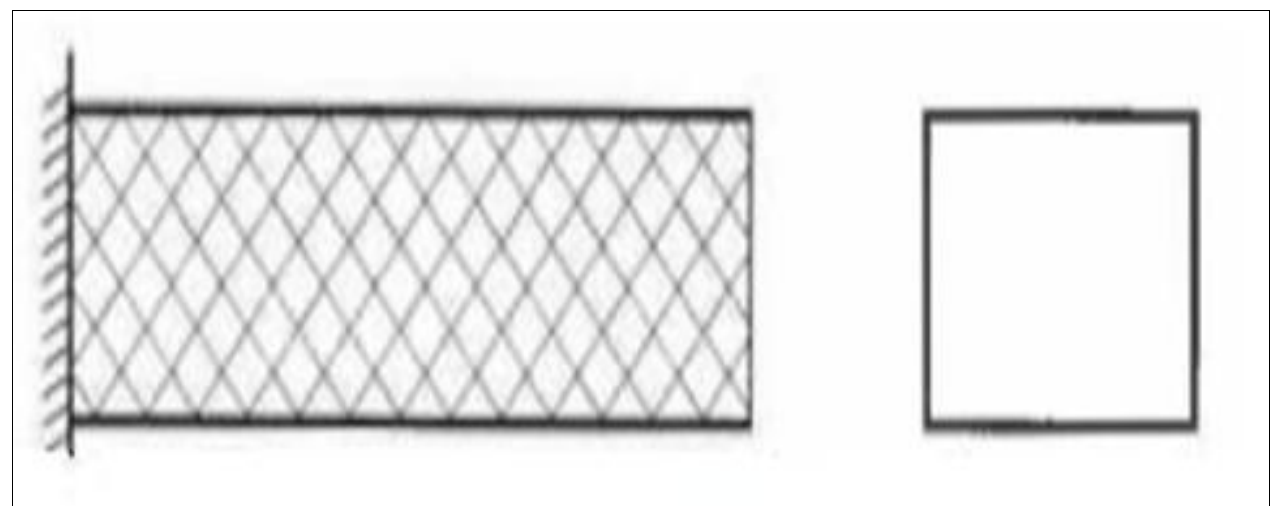

Fig. 2 Fiber orientation for handling shear due to bending.

The strength of the composite is determined by matrix if the force applied is perpendicular to the fiber and fibers and matrix determines its stiffness. For force applied parallel to the fibers; the stiffness as well as strength of the composite is determined by the fibers.

K. Al-Khazaraji et al. [3] studied fatigue and tensile characteristics of carbon fiber for prosthetic foot using different types of reinforcements. They conducted tensile and fatigue tests using ANSYS-11 and compared the values with the theoretical results. They compared properties of 5 different reinforcements with epoxy resin i.e. glass fibers, Perlon fiber, (carbon + glass) fiber, carbon fiber, (carbon + glass) fibers $+\mathrm{SiO} 2$ particles. The result shows that the mechanical properties of the carbon fiber can be improved by changing the type of reinforcement. The scientists concluded that ( epoxy + carbon reinforcement) composite gives better mechanical properties i.e. improved fatigue strength due to high value of Young's modulus and also higher factor of safety is obtained whereas Perlon reinforcement gave lowest values in all properties. NamahHadi et al. [4] studied short carbon fiber blend (5-15\%) reinforced with PolyMethyl Metha Acrylate (PMMA) and Silicone rubber (SR). Authors experimented these two materials so as to improve flexural strength of carbon fiber. They prepared different polymer blends by changing the concentration ratio of (PMMA: SR) from 90:10, 80:20, 70:30, 60:40 to 50:50. These blends also support dorsiflexion movement of ankle foot. The result of the test for flexural strength concluded that flexural strength of polymer blends increases as reinforcement with carbon fiber increases from 5-15\%. (PMMA: SR) (80:20) reinforced with 15\% carbon fiber improved the flexural strength by approximately $17 \%$, giving the highest value of all. The author concluded that polymer blends reinforced with short carbon fiber will not only increase flexural strength of material but also the flexural modulus, reducing the cost.

\section{Kevlar}

Berry et al. [2] studied and compared mechanical properties of Carbon Fiber, Kevlar and Glass Fiber. Authors analyzed that Kevlar is the lightest material compared with Carbon Fiber and Fiber Glass and is also very expensive. It gives excellent fracture resistance under fracture loading and can also absorb high loads of stress and torque. However, Kevlar is weak in maintaining structure under load and is about five times weak under compression than under tension. Tensile strength of Kevlar is $15^{*} 10^{\wedge} 3$ psi whereas its compressive strength is $3^{*} 10^{\wedge} 3$ psi. Kevlar gives excellent chemical resistance. It is difficult to saturate Kevlar with resin. Hence to overcome this Author blend Kevlar with carbon fiber producing a quasi iso-trophic hybrid by arranging composite fibers in mat/knit form. This places fibers in three dimensional or multiplane manner. This hybrid blend improved drawbacks of Kevlar desirably providing lightness, stiffness as well as torque and impact resistance.

\section{Fiber Glass}

Berry et al. [2] studied properties of Fiber Glass. It was found that Fiber Glass is easy to saturate with resin. It is also possible to obtain it in different quality and forms. It is highly durable and flexible. Its fiber strength is twice stronger under tension than under compression. Its tensile strength is $10^{*} 10^{\wedge} 3$ psi whereas its compressive strength is $5^{*} 10^{\wedge} 3$ psi. The material is also cost efficient. But the high flexibility of the material makes it difficult to regain its original shape after the toe off and hence doesn't provide enough momentum.

Authors then studied blend of Fiber Glass with Carbon Fiber which achieved better properties. Hybrid of Carbon Fiber and Fiber Glass can achieve good resistance to fracture along with good strength to weight ratio. The hybrid shows properties of Carbon Fiber as well as Fiber Glass i.e. stiffness, lightweight, durable, flexible as well as cost efficient. 


\section{Result And Discussion}

The results shown by study of above material are compared.

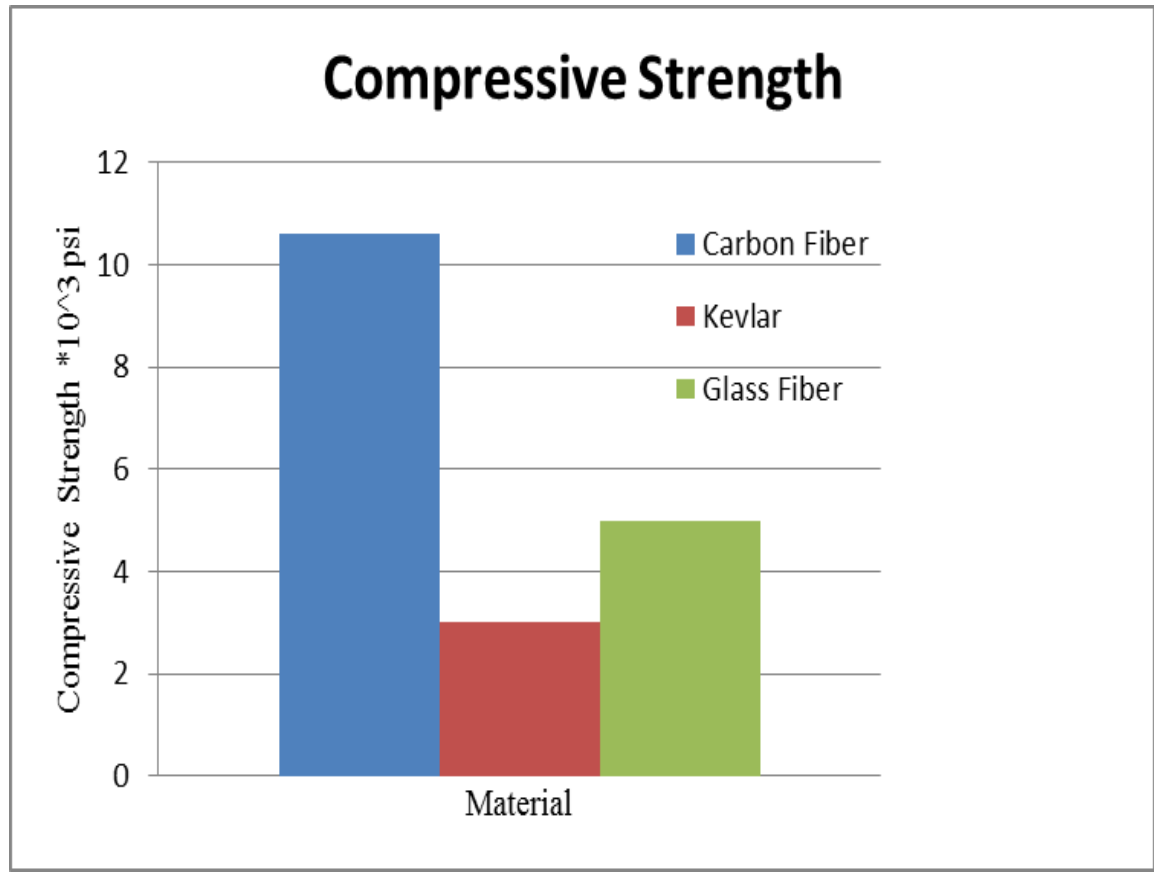

Fig. 3 compressive strength v/s Material

From Fig 3, we can conclude that Kevlar has least compressive strength $\left(3 * 10^{\wedge} 3 \mathrm{psi}\right)$ as compared with Carbon Fiber and Glass Fiber, whereas Carbon Fiber gives the highest value of compressive strength i.e. $\left(10.6^{*} 10^{\wedge} 3 \mathrm{psi}\right)$. Glass Fiber gives moderate value of compressive strength $\left(5 * 10^{\wedge} 3 \mathrm{psi}\right)$.

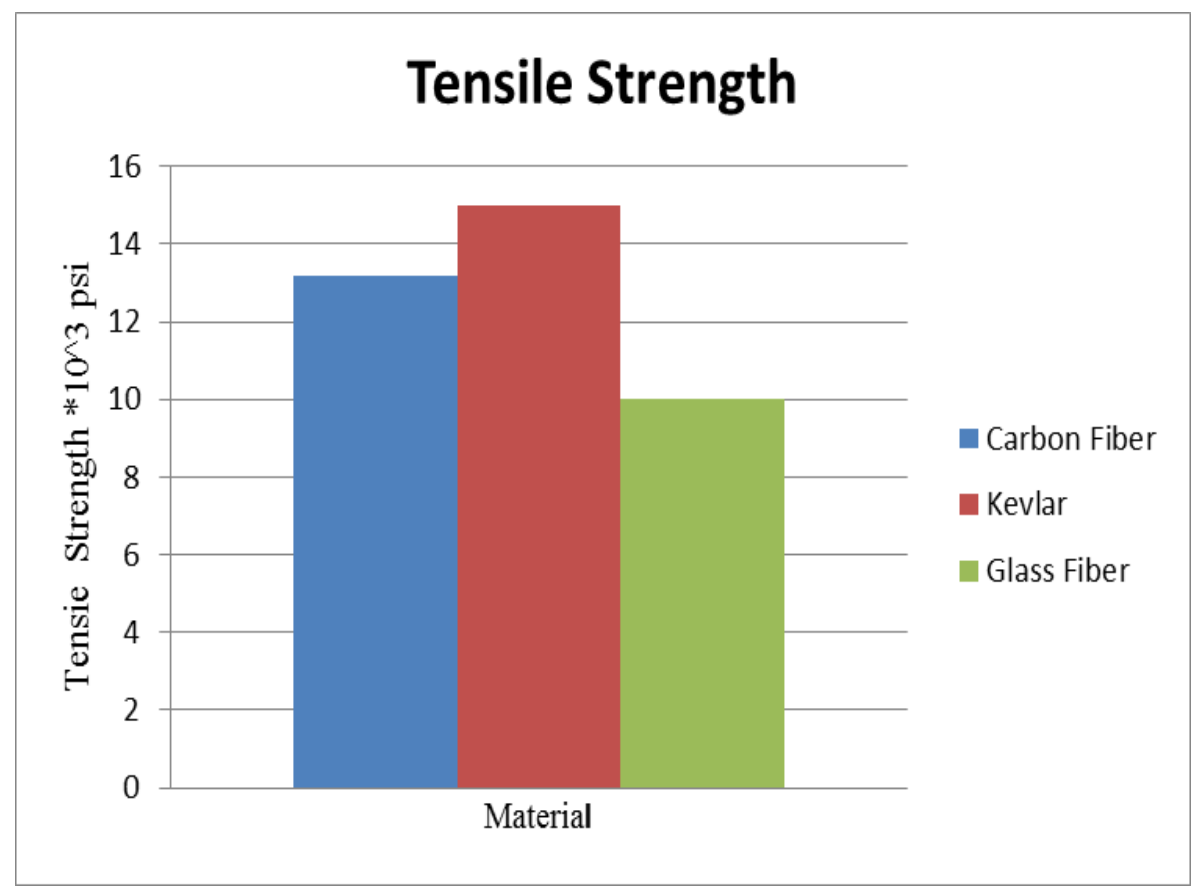

Fig. 4 Tensile Strength vs Material

Fig. 4 concludes that Kevlar has the highest value of tensile strength $\left(15^{*} 10^{\wedge} 3\right.$ psi) and Glass Fiber gives least value $\left(10^{*} 10^{\wedge} 3 \mathrm{psi}\right)$, whereas Carbon Fiber gives moderate value $\left(13.2^{*} 10^{\wedge} 3 \mathrm{psi}\right)$. 


\section{The conclusions of the literature review are as follows:}

\section{Conclusion}

- It is observed that orientation of fibers decides the properties of material hence the mechanical properties of Carbon Fibers as well as Glass Fiber can be improved by adapting the sandwich structure of orientation of fiber laminates.

- Blending of Carbon Fiber with PolyMethyl Metha Acrylate (PMMA) and Silicone rubber (SR) increases its flexural strength by almost $17 \%$.

- The type of reinforcement also determines the material properties. Hence blending of Carbon Fiber with Glass Fiber will not only improve its mechanical properties but also reduce its cost and increase its durability.

- Hybrid blend of Carbon Fiber with Kevlar also shows excellent improvement in mechanical properties as lightweight, stiffness, high tensile as well as compressive strength, desired flexibility and also cost efficient. Hence by considering the body weight of the amputee, gait, and analyzing the material properties; best suitable material should be chosen.

\section{References}

[1]. B. L. Klasson, "Carbon fiber and fibril lamination in prosthetics and orthotics: some basic theory and practical advice for the practitioner ", Prosthetics and Orthotics International, Vol. 19, 1995, 74-91.

[2]. D. A. Berry and C.P.(C),"Composite Materials for Orthotics and Prosthetics", Orthotics and Prosthetics, vol. 40(4) 1987, pp. 35-43.

[3]. K. Al-Khazraji, J. Kadhim and P. S. Ahmed, "Tensile and Fatigue Characteristics of Lower-Limb Prosthetic Socket Made from Composite Materials ", International Conference on Industrial Engineering and Operations Management Istanbul, Turkey, 2012.

[4]. A. NamahHadi and J. Oleiwi, "Improve Flexural Strength of PMMA/SR Polymer Blend by Reinforcement with Carbon Fibers as Prosthetic Foot Polymer Material"; International Journal of Application or Innovation in Engineering \& Management (IJAIEM),Vol. 4(2), 2015 ,ISSN 2319-4847

[5]. R. C. Me, R. Ibrahim and P. Tahir, "Natural based biocomposite material for prosthetic socket fabrication"

[6]. "Prosthetics -Testing of ankle- foot devices and foot units - Requirements and test methods", ISO 22675, vol.2, 2016.

[7]. G. McGimpsey and C. Terry,"Limb Prosthetics Services and Devices Critical Unmet Need: Market Analysis "

[8]. B. Stier, J. Simon and S. Reese, "Numerical and experimental investigation of the structural behavior of a carbon fiber reinforced ankle-foot orthosis"; Elsevier Ltd. 2015, vol. 37(5), pp. 505-11 